\title{
Generalized Parametric Solutions to Multiple Sums of Powers
}

\author{
Oliver Couto ${ }^{1, *}$, Seiji Tomita ${ }^{2}$ \\ ${ }^{1}$ Department of Mathematics, University of Waterloo, Canada \\ ${ }^{2}$ Tokyo Software Company (Inc.), Japan
}

Copyright $(\mathcal{C} 2015$ by authors, all rights reserved. Authors agree that this article remains permanently open access under the terms of the Creative Commons Attribution License 4.0 International License

\begin{abstract}
The work on equation (B) below has been studied by others for different degree ' $n$ ' and equation (A) below for degree five, has been previously published by Mr. Ajai Choudhry (Ref. no. 1). But combined systematic analysis for degrees $2,3,4,5,6,7,8$ \& 9 etc. has not been done before, as is done in this paper. Consider the below mentioned equations: $a s^{n}+b t^{n}+c u^{n}=a w^{n}+b x^{n}+c y^{n}---(A)$ and $a s^{n}+b t^{n}+c u^{n}+d v^{n}=a w^{n}+b x^{n}+c y^{n}+d z^{n}--(B)$
\end{abstract}

Keywords Sums of Powers, Diophantine Equations, Number Theory, Pure Math

\section{Introduction}

The equation (A) in this paper has three terms on either side in section one and equation (B) has four terms on both sides in section two. In other words this paper deals with degree's $(2,3,4,5,6,7 \& 8)$ for equation (B) \& gives a generalized parametric solutions for any degree ' $n$ ' for equation (A). As is known that solving Diophantine equations for power five and above is quite difficult. In this paper we show that equation (A) given above is solvable when ( $\mathrm{s}, \mathrm{t}, \mathrm{u}, \mathrm{w}, \mathrm{x}, \mathrm{y})$ are known and we provide methods to find integer coefficents $(a, b, c)$ for which the above Diophantine equation is satisfied for degree $<10$. Similarly, analysis is provided for equation (B) also. This paper thus shows that parametric solutions are possible for the Diophantine equations (B) for degree $\mathrm{n}<10$ \& gives a generalized parametric solution for equation (A) above for any degree 'n'.

\section{Discussion (See Below)}

\section{Consider Equation}

Let $\mathrm{S}=\mathrm{pt}+1, \mathrm{~T}=\mathrm{qt}+1, \mathrm{U}=\mathrm{t}+1$

$$
a S^{n}+b T^{n}+c U^{n}=a W^{n}+b X^{n}+c Y^{n}
$$

and $\mathrm{W}=\mathrm{pt}-1, \mathrm{X}=\mathrm{qt}-1, \mathrm{Y}=\mathrm{t}-1$

\& $\quad c=-(a p+b q)$

After substituting in equation (A) and solving for the coefficients (a,b,c) we get:

For even degree's $(n=4,6 \& 8)$

$\mathrm{n}=4$ we get :

$$
\begin{gathered}
a=-\left(2 t^{2}\left(q^{3}-1\right)+2(q-1)\right) \\
b=\left(2 t^{2}\left(p^{3}-1\right)+2(p-1)\right) \\
c=-\left(2 t^{2}\left(p^{3}-q^{3}\right)+2(p-q)\right)
\end{gathered}
$$

For $n=6$ we get:

$$
\begin{gathered}
a=-3 t^{4}\left(q^{5}-1\right)-10 t^{2}\left(q^{3}-1\right)-3(q-1) \\
b=3 t^{4}\left(p^{5}-1\right)+10 t^{2}\left(p^{3}-1\right)+3(p-1) \\
c=-3 t^{4}\left(p^{5}-q^{5}\right)-10 t^{2}\left(p^{3}-q^{3}\right)-3(p-q)
\end{gathered}
$$


For $\mathrm{n}=8$ we get:

$$
\begin{gathered}
a=-\left(4 t^{6}\left(q^{7}-1\right)+28 t^{4}\left(q^{5}-1\right)+28 t^{2}\left(q^{3}-1\right)+4(q-1)\right) \\
b=\left(4 t^{6}\left(q^{7}-1\right) 28 t^{4}\left(p^{5}-1\right)+28 t^{2}\left(p^{3}-1\right)+4(p-1)\right) \\
c=-\left(4 t^{6}\left(p^{7}-q^{7}\right)+28 t^{4}\left(p^{5}-q^{5}\right)+28 t^{2}\left(p^{3}-q^{3}\right)+4(p-q)\right)
\end{gathered}
$$

Note: Similarly for $n=10,12,14$, etc. similar pattern with the coefficient's is given in the table below.

\section{For odd degree's $(n=3,5,7 \& 9)$}

$\mathrm{n}=3$ we get

$$
\begin{gathered}
a=-\left(3 t^{2} q(q-1)-(q-1)\right) \\
b=\left(3 t^{2} p(p-1)-(p-1)\right) \\
c=-\left(3 t^{2} p q(p-q)-(p-q)\right)
\end{gathered}
$$

For $\mathrm{n}=5$ we get,

$$
\begin{gathered}
a=-\left(5 t^{4} q\left(q^{3}-1\right)+10 t^{2} q(q-1)-(q-1)\right) \\
b=\left(5 t^{4} p\left(p^{3}-1\right)+10 t^{2} p(p-1)-(p-1)\right) \\
c=-\left(5 t^{4} p q\left(p^{3}-q^{3}\right)+310 p q(p-q)-(p-q)\right)
\end{gathered}
$$

For $\mathrm{n}=7$ we get,

$$
\begin{gathered}
a=-\left(7 t^{6} q\left(q^{5}-1\right)+35 t^{4} q\left(q^{3}-1\right)+21 t^{2} q(q-1)-(q-1)\right) \\
b=\left(7 t^{6} q\left(q^{5}-1\right) 35 t^{4} p\left(p^{3}-1\right)+21 t^{2} p(p-1)-(p-1)\right) \\
c=-\left(7 t^{6} p q\left(p^{5}-q^{5}\right)+35 t^{4} p q\left(p^{3}-q^{3}\right)+21 t^{2} p q(p-q)-(p-q)\right)
\end{gathered}
$$

For $\mathrm{n}=9$ we get,

$$
\begin{gathered}
a=-\left(9 t^{8} q\left(q^{7}-1\right)+84 t^{6} q\left(q^{5}-1\right)+126 t^{4} q\left(q^{3}-1\right)+36 t^{2} q(q-1)-(q-1)\right) \\
\left.b=\left(9 t^{8} * p\left(p^{7}-1\right)+84 t^{6} * p\left(p^{5}-1\right)+126 t^{\wedge} 4 p\left(p^{3}-1\right)+36 t\right) \wedge 2 p(p-1)-(p-1)\right) \\
c=-\left(9 t^{8} * p q\left(p^{7}-q^{7}\right)+84 t^{6} p q\left(p^{5}-q^{5}\right)+126 t^{4} p q\left(p^{3}-q^{3}\right)+36 t^{2} p q(p-q)-(p-q)\right)
\end{gathered}
$$

Note: Similarly for odd ' $n$ ' $=11,13,15$, etc a similar pattern of the coefficient's is given, in the attached tables.

Table 1. Pascal's Triangle

\begin{tabular}{|l|l|l|l|l|l|l|l|l|l|l|l|l|l|l|l|l|l|l|l|l|l|}
\hline $\mathrm{n}$ & & $a^{*}$ & $\mathrm{~b}$ & $\mathrm{c}$ & $\mathrm{d}$ & $\mathrm{e}$ & $\mathrm{f}$ & $\mathrm{g}$ & $\mathrm{h}$ & $\mathrm{j}$ & $\mathrm{k}$ & $\mathrm{L}$ & $\mathrm{m}$ & $\mathrm{n}$ & $\mathrm{p}$ & $\mathrm{q}$ & $\mathrm{r}$ & $\mathrm{s}$ & $\mathrm{t}$ & $\mathrm{u}$ & \\
\hline & & & & & & & & & & & & & & & & & & & & \\
\hline & & & & & & & & & & & 1 & & & & & & & & & \\
\hline 1 & & & & & & & & & & 1 & & 1 & & & & & & & & & \\
\hline 2 & & & & & & & & & 1 & & 2 & & 1 & & & & & & & \\
\hline 3 & & & & & & & & 1 & & 3 & & 3 & & 1 & & & & & & \\
\hline 4 & & & & & & & 1 & & 4 & & 6 & & 4 & & 1 & & & & & & \\
\hline 5 & & & & & & 1 & & 5 & & 10 & & 10 & & 5 & & 1 & & & & \\
\hline 6 & & & & & 1 & & 6 & & 15 & & 20 & & 15 & & 6 & & 1 & & & \\
\hline 7 & & & & 1 & & 7 & & 21 & & 35 & & 35 & & 21 & & 7 & & 1 & & \\
\hline 8 & & & 1 & & 8 & & 28 & & 56 & & 70 & & 56 & & 28 & & 8 & & 1 & \\
\hline 9 & & 1 & & 9 & & 36 & & 84 & & 126 & & 126 & & 8 & & 36 & & 9 & & 1 \\
\hline
\end{tabular}




\section{We have the well known Pascal's Triangle (given above)}

Above is for different degree's ' $n$ ':

Above is table of Coefficient's of expansion of $(m+1)^{\wedge} n$

*Coefficient ( $\mathrm{a}, \mathrm{b}, \mathrm{c}, \mathrm{d}, \mathrm{e}, \mathrm{f},--------\mathrm{u})$ for $\left(\mathrm{m}^{p}\right)$ and selected degree ' $\mathrm{n}$ ' is given by,

$$
C_{p}^{n}=\frac{n !}{[p ! *(n-p) !]}
$$

Where ( $n$ !) means ' $n$ ' factorial

(See below for more tables)

Equation,

$$
a S^{n}+b T^{n}+c U^{n}=a W^{n}+b X^{n}+c Y^{n}
$$

Table 2. For odd degree

\begin{tabular}{|c|c|c|c|c|c|c|c|c|c|c|}
\hline Degree $\mathrm{n}$ & constant & $\begin{array}{c}\text { Coeff. } \\
\left(t^{2}\right)\end{array}$ & $\begin{array}{c}\text { Coeff. } \\
\left(t^{4}\right)\end{array}$ & Coeff. $\left(t^{6}\right)$ & $\begin{array}{c}\text { Coeff. } \\
\left(t^{8}\right)\end{array}$ & $\begin{array}{c}\text { Coeff. } \\
\left(t^{10}\right)\end{array}$ & $\begin{array}{c}\text { Coeff. } \\
\left(t^{12}\right)\end{array}$ & $\begin{array}{c}\text { Coeff. } \\
\left(t^{14}\right)\end{array}$ & $\begin{array}{c}\text { Coeff. } \\
\left(t^{16}\right)\end{array}$ & Remarks \\
\hline 3 & 1 & $\mathrm{n}$ & - & - & - & - & & & & \\
\hline 5 & 1 & $2 \mathrm{n}$ & $\mathrm{n}$ & - & - & - & & & & \\
\hline 7 & 1 & $3 \mathrm{n}$ & $5 \mathrm{n}$ & $\mathrm{n}$ & - & - & & & & $\mathrm{n}=\mathrm{prime}$ \\
\hline 9 & 1 & $4 \mathrm{n}$ & $14 \mathrm{n}$ & 84 & $\mathrm{n}$ & - & & & & \\
\hline 11 & 1 & $5 \mathrm{n}$ & $30 \mathrm{n}$ & $42 \mathrm{n}$ & $15 \mathrm{n}$ & $\mathrm{n}$ & & & & \\
\hline 13 & 1 & $6 \mathrm{n}$ & $55 \mathrm{n}$ & $132 \mathrm{n}$ & $99 \mathrm{n}$ & $22 \mathrm{n}$ & $\mathrm{n}$ & & & $\mathrm{n}$ \\
\hline 15 & 1 & $7 \mathrm{n}$ & $91 \mathrm{n}$ & 5005 & $429 \mathrm{n}$ & 3003 & 455 & $\mathrm{n}$ & $\mathrm{n}=\mathrm{prime}$ \\
\hline 17 & 1 & $8 \mathrm{n}$ & $140 \mathrm{n}$ & $728 \mathrm{n}$ & $1430 \mathrm{n}$ & $1144 \mathrm{n}$ & $364 \mathrm{n}$ & $40 \mathrm{n}$ & $\mathrm{n}$ & \\
\hline
\end{tabular}

Where $(\mathrm{a}, \mathrm{b}, \mathrm{c})$ are polynomials in $(\mathrm{t})$

For above table, coefficients of $\left(t^{2}\right)$ is given by, $=n(n-1) / 2$

For degree eleven we get coefficient of $\left(t^{2}\right)=n *(11-1) / 2=5 n$

and the coefficients of $\left(t^{4}\right)$ is given by $=n(n-1)(n-2)(n-3) / 24$

For degree eleven we get coeff. of $\left(t^{4}\right)=n^{*}(11-1) *(11-2) *(11-3) / 24=30 n$

Table 3. For degree ' $n$ ' even

\begin{tabular}{|c|c|c|c|c|c|c|c|}
\hline Degree $\mathrm{n}$ & constant & Coeff. ( $\left.\mathrm{t}^{\wedge} 2\right)$ & $\begin{array}{c}\text { Coeff. } \\
\left(\mathrm{t}^{\wedge} 4\right)\end{array}$ & Coeff. $\left(\mathrm{t}^{\wedge} 6\right)$ & Coeff. ( $\left.\mathrm{t}^{\wedge} 8\right)$ & Coeff. ( $\left.\mathrm{t}^{\wedge} 10\right)$ & ${\text { Coeff. }\left(\mathrm{t}^{\wedge} 12\right)}$ \\
\hline 4 & 2 & 2 & & & & & \\
\hline 6 & 3 & 10 & 3 & & & & \\
\hline 8 & 4 & 28 & 28 & 4 & & & \\
\hline 10 & 5 & 60 & 126 & 60 & 5 & & \\
\hline 12 & 6 & 110 & 396 & 396 & 110 & 6 & \\
\hline 14 & 7 & 182 & 1001 & 1716 & 1001 & 182 & 7 \\
\hline
\end{tabular}

The constant terms in above table has the relation given below Table (3),

$$
\mathrm{K}=(\mathrm{n})(\mathrm{n}-1)(\mathrm{n}-2) / 12 \text { for degree } 12
$$

For degree $n=12$, the coefficient of constant term is $=(12 * 11 * 10) / 12=110$ 
Table 4. For odd degree ' $n$ '

\begin{tabular}{|c|c|c|}
\hline $\begin{array}{l}\text { Coefficient's for polynomial (a) in } \\
\text { equation (A) above }\end{array}$ & Representation for degree 'n' $=(3,5,7,9,-------u)$ & Remarks \\
\hline$t^{0}$ & 1 & \\
\hline$t^{2}$ & $\mathrm{n} *(\mathrm{n}-1) /(2 !)$ & \\
\hline$t^{4}$ & $\mathrm{n}(\mathrm{n}-1)(\mathrm{n}-2)(\mathrm{n}-3) /(4 !)$ & \\
\hline$t^{6}$ & $\mathrm{n}(\mathrm{n}-1)(\mathrm{n}-2)(\mathrm{n}-3)(\mathrm{n}-4)(\mathrm{n}-5) /(6 !)$ & \\
\hline $\mathrm{t}^{\wedge} \mathrm{p},(\mathrm{p}=8)$ & $\mathrm{n}(\mathrm{n}-1)(\mathrm{n}-2)(\mathrm{n}-3)(\mathrm{n}-4)(\mathrm{n}-5)(\mathrm{n}-6)(\mathrm{n}-\mathrm{p}+1) /(\mathrm{p} !)$ & \\
\hline--- & ---------------- & \\
\hline 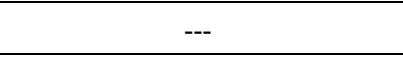 & 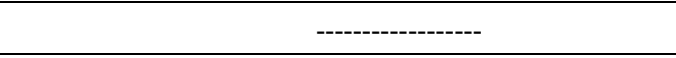 & \\
\hline--- & ---------------- & \\
\hline$t^{p}$ & $=\frac{n !}{[(p !)(n-p) !]}$ & $\begin{array}{l}\text { Same formula as Pascal's } \\
\text { table } \\
\qquad C_{p}^{n}\end{array}$ \\
\hline
\end{tabular}

Where, ( $p$ !), represents (p) factorial

Table 5. For even degree ' $n$ '

\begin{tabular}{|c|c|c|}
\hline $\begin{array}{c}\text { Coeeficent's for polynomial } \\
\text { (a) in equation (A) } \\
\text { above } \\
\end{array}$ & Representation for degree ' $\mathrm{n}$ ' $=(4,6,8,10,-------\mathrm{v})$ & Remarks \\
\hline$t^{0}$ & $2 n(n-1)(n-2) /(4 !)$ & \\
\hline$t^{2}$ & $3 n(n-1)(n-2)(n-3)(n-4) /(6 !)$ & \\
\hline$t^{4}$ & $4 n(n-1)(n-2)(n-3)(n-4)(n-5)(n-6) /(8 !)$ & \\
\hline$t^{6}$ & $5 n(n-1)(n-2)(n-3)(n-4)(n-5)(n-6)(n-7)(n-8) /(10 !)$ & \\
\hline$t^{p}(\mathrm{p}=8)$ & {$[(\mathrm{p}+4)(\mathrm{n})(\mathrm{n}-1)(\mathrm{n}-2)(\mathrm{n}-3)(\mathrm{n}-4)(\mathrm{n}-5)(\mathrm{n}-6)(\mathrm{n}-\mathrm{p}-2) / 2] *((\mathrm{p}+4) !)$} & \\
\hline-- & -------------- & \\
\hline--- & -------------- & \\
\hline--- & --------------- & \\
\hline$t^{p}$ & $=\frac{n !}{[(w) !(n-w) !]}$ & $\begin{array}{l}\text { This representation is similar like } \\
\text { the form given in table (a) above. } \\
\qquad C_{w}^{n}\end{array}$ \\
\hline
\end{tabular}

Where, $(\mathrm{w}$ ! ) represents $[(\mathrm{p}+1)$ ! ] factorial \& ' $\mathrm{n}$ ' is relevant degree of the equation.

For $\mathrm{n}=9$ we get the following equations for the coefficients $(\mathrm{a}, \mathrm{b}, \mathrm{c})$ of the equation $(\mathrm{A})$ and is given below,

$$
\begin{gathered}
a=-\left(C_{8}^{n} * t^{8} q\left(q^{7}-1\right)+C_{6 *}^{n} t^{6} q\left(q^{5}-1\right)+C_{4 *}^{n} t^{4} q\left(q^{3}-1\right)+C_{2 *}^{n} t^{2} q(q-1)-C_{0 *}^{n}(q-1)\right) \\
b=\left(C_{8 *}^{n} t^{8} * p\left(p^{7}-1\right)+C_{6}^{n} * t^{6} * p\left(p^{5}-1\right)+C_{4}^{n} * t^{4} p\left(p^{3}-1\right)+C_{2 *}^{n} t^{\wedge} 2 p(p-1)-C_{0 *}^{n}(p-1)\right) \\
c=-\left(C_{8 *}^{n} t^{8} * p q\left(p^{7}-q^{7}\right)+C_{6 *}^{n} t^{6} p q\left(p^{5}-q^{5}\right)+C_{4}^{n} * t^{4} p q\left(p^{3}-q^{3}\right)+C_{2 *}^{n} t^{2} p q(p-q)-C_{0 *}^{n}(p-q)\right)
\end{gathered}
$$

Putting $\mathrm{n}=9$ we get,

$$
\begin{aligned}
a= & -\left(C_{8}^{n} * t^{8} q\left(q^{7}-1\right)+C_{6 *}^{n} t^{6} q\left(q^{5}-1\right)+C_{4 *}^{n} t^{4} q\left(q^{3}-1\right)+C_{2 *}^{n} t^{2} q(q-1)-C_{0 *}^{n}(q-1)\right) \\
& =-\left(9 t^{8} q\left(q^{7}-1\right)+84 t^{6} q\left(q^{5}-1\right)+126 t^{4} q\left(q^{3}-1\right)+36 t^{2} q(q-1)-(q-1)\right)
\end{aligned}
$$

Similarly we get:

$$
\begin{gathered}
b=\left(9 t^{8} * p\left(p^{7}-1\right)+84 t^{6} * p\left(p^{5}-1\right)+126 t^{\wedge} 4 p\left(p^{3}-1\right)+36 t^{2} p(p-1)-(p-1)\right) \\
c=-\left(9 t^{8} * p q\left(p^{7}-q^{7}\right)+84 t^{6} p q\left(p^{5}-q^{5}\right)+126 t^{4} p q\left(p^{3}-q^{3}\right)+36 t^{2} p q(p-q)-(p-q)\right)
\end{gathered}
$$

\section{General equation for degree ' $\mathbf{n}$ ' odd is given below,}

$a=-\left(C_{(n-1)}^{n} * t^{(n-1)} q\left(q^{(n-2)}-1\right)+C_{(n-3) *}^{n} t^{(n-3)} q\left(q^{(n-4)}-1\right)+C_{(n-5) *}^{n} t^{(n-5)} q\left(q^{(n-6)}-1\right)+------\right.$ $\left.----+C_{2 *}^{n} t^{2} q(q-1)-C_{0 *}^{n}(q-1)\right)$

And similarly for $(\mathrm{b} \& \mathrm{c})$ 
For even, $\mathrm{n}=8$ we get:

$$
\begin{gathered}
a=\left(-\frac{1}{2}\right) *\left(C_{w}^{n *} t^{6}\left(q^{7}-1\right)+C_{w}^{n *} t^{4}\left(q^{5}-1\right)+---+\right. \\
\left.C_{w}^{n *} t^{2}\left(q^{3}-1\right)+C_{w}^{n *}(q-1)\right) \\
b=\left(-\frac{1}{2}\right) *\left(8 t^{6}\left(q^{7}-1\right)+56 t^{4}\left(p^{5}-1\right)+56 t^{2}\left(p^{3}-1\right)+8(p-1)\right) \\
c=\left(-\frac{1}{2}\right) *\left(8 t^{6}\left(p^{7}-q^{7}\right)+56 t^{4}\left(p^{5}-q^{5}\right)+56 t^{2}\left(p^{3}-q^{3}\right)+8(p-q)\right)
\end{gathered}
$$

Where $(w=p+1)$

\section{Hence general solution for even degree ' $n$ ' is given below,}

$$
\begin{gathered}
a=\left(-\frac{1}{2}\right) *\left(C_{(n-1)}^{n} * t^{(n-2)}\left(q^{(n-1)}-1\right)+C_{(n-3)}^{n} * t^{(n-4)}\left(q^{(n-3)}-1\right)+-------\right. \\
\left.+C_{3}^{n *} t^{2}\left(q^{3}-1\right)+C_{1}^{n *}(q-1)\right)
\end{gathered}
$$

For example in the above for $\mathrm{n}=8$ \& coefficient of $\left(t^{\wedge} p\right)=\left(t^{\wedge} 2\right)$ we get for $\mathrm{p}=2$. $w=p+1=2+1=3$,

\begin{tabular}{|c|c|c|c|c|c|c|c|c|c|}
\hline Degree 'n' & $\mathrm{a}$ & $\mathrm{b}$ & $\mathrm{c}$ & $\mathrm{S}$ & $\mathrm{t}$ & $\mathrm{u}$ & $\mathrm{w}$ & $\mathrm{x}$ & $\mathrm{y}$ \\
\hline 3 & -23 & 70 & -71 & 7 & 5 & 3 & 5 & 3 & 1 \\
\hline 5 & -1199 & 6478 & -9359 & 7 & 5 & 3 & 5 & 3 & 1 \\
\hline 7 & -35783 & 369430 & -631511 & 7 & 5 & 3 & 5 & 3 & 1 \\
\hline
\end{tabular}

$$
\begin{aligned}
& C_{w}^{n}=C_{3}^{8}=\frac{(8 * 7 * 6 * 5 * 4 * 3 * 2 * 1)}{(5 * 4 * 3 * 2 * 1) *(3 * 2 * 1)}=56 \\
& a=\left(-\frac{1}{2}\right) *\left(C_{7}^{n *} t^{6}\left(q^{7}-1\right)+C_{5}^{n *} t^{4}\left(q^{5}-1\right)+\right. \\
& \left.C_{5}^{n *} t^{2}\left(q^{3}-1\right)+C_{1}^{n *}(q-1)\right) \\
& =\left(-\frac{1}{2}\right) *\left(8^{*} t^{6}\left(q^{7}-1\right)+56^{*} t^{4}\left(q^{5}-1\right)+\right. \\
& \left.56 * t^{2}\left(q^{3}-1\right)+8 *(q-1)\right) \\
& =-\left(4^{*} t^{6}\left(q^{7}-1\right)+28 * t^{4}\left(q^{5}-1\right)+28 * t^{2}\left(q^{3}-1\right)+4 *(q-1)\right)
\end{aligned}
$$

Similarly we get,

$$
\begin{gathered}
b=\left(4 t^{6}\left(q^{7}-1\right) 28 t^{4}\left(p^{5}-1\right)+28 t^{2}\left(p^{3}-1\right)+4(p-1)\right) \\
c=-\left(4 t^{6}\left(p^{7}-q^{7}\right)+28 t^{4}\left(p^{5}-q^{5}\right)+28 t^{2}\left(p^{3}-q^{3}\right)+4(p-q)\right)
\end{gathered}
$$

Numerical solutions by taking values of variable $(\mathrm{p}, \mathrm{q}, \mathrm{t})=(3,2,2)$ is given in the below table

$$
\begin{aligned}
& (s, t, u, v)=(p t+1, q t+1, r t+1, t+1) \\
& (w, x, y, z)=(p t-1, q t-1, r t-1, t-1)
\end{aligned}
$$

Table 6. Below for odd degree 'n' find numerical solutions

Section (2)

$$
\begin{gathered}
a s^{n}+b t^{n}+c u^{n}+d v^{n}=a w^{n}+b x^{n}+c y^{n}+d z^{n} \\
\text { Let }(s, t, u, v)=(p m+1, q m+1, r m+1, m+1) \\
(w, x, y, z)=(p m-1, q m-1, r m-1, m-1)
\end{gathered}
$$




\section{For degree two:}

From equation $(B)$ for $n=2$ we have,

$$
a s^{2}+b t^{2}+c u^{2}+d v^{2}=a w^{2}+b x^{2}+c y^{2}+d z^{2}
$$

Substituting \& simplifying we get,

$$
\begin{gathered}
a\left(s^{2}-w^{2}\right)+b\left(t^{2}-x^{2}\right)+c\left(u^{2}-y^{2}\right)+d\left(v^{2}-z^{2}\right)=0 \\
\text { and we get the condition, } a p+b q+c r+d=0
\end{gathered}
$$

The above has solution

$$
(a, b, c, d)=(1,1,1,-9) \&(p, q, r)=(4,3,2)
$$

For $m=2$ in equation $(C) \&(D)$ we get

$$
\begin{aligned}
& (s, t, u, v)=(9,7,5,3) \\
& (w, x, y, z)=(7,5,3,1)
\end{aligned}
$$

Hence we get:

$$
\begin{gathered}
1(9)^{2}+1(7)^{2}+1(5)^{2}+(-9)(3)^{2}= \\
1(7)^{2}+1(5)^{2}+1(3)^{2}+(-9)(1)^{2}
\end{gathered}
$$

\section{Degree Three :}

$$
\begin{gathered}
a s^{3}+b t^{3}+c u^{3}+d v^{3}=a w^{3}+b x^{3}+c y^{3}+d z^{3}--(\mathrm{B}) \\
\text { Let }(s, t, u, v)=(p m+1, q m+1, r m+1, m+1)---(\mathrm{C}) \\
(w, x, y, z)=(p m-1, q m-1, r m-1, m-1)---(\mathrm{D})
\end{gathered}
$$

Substituting \& simplifying we get :

$$
\begin{gathered}
a\left(s^{3}-w^{3}\right)+b\left(t^{3}-x^{3}\right)+c\left(u^{3}-y^{3}\right)+d\left(v^{3}-z^{3}\right)=0 \\
\left(6 d+6 a p^{2}+6 b q^{2}+6 c r^{2}\right) m^{2}+2 a+2 b+2 c+2 d=0
\end{gathered}
$$

Hence we have to find rational number

$\{a, b, c, d, p, q, r, h\}$.

$$
\begin{gathered}
h^{2}=-12 p^{2} a^{2}+\left(\left(-12 q^{2}-12 p^{2}\right) b-12 d-12 c r^{2}-12 p^{2}(c+d)\right) a \\
-12 q^{2} b^{2}+\left(-12 d-12 c r^{2}-12 q^{2}(c+d)\right) b-12\left(d+c r^{2}\right)(c+d)
\end{gathered}
$$

Let use a known solution $[p, q, r]=[4,3,2]$,

$$
[a, b, c, d]=[1,2,6,-10], h=[24], m=1 / 12
$$

Substitute

the above to $(C) \&(D)$, then we get a parametric solution as follows.

$$
\begin{gathered}
{[a, b, c, d]=\left[k^{\wedge} 2-48 k-504\right.} \\
\left.2 k^{2}-48 k+936,6 k^{2}-48 k+6696,-10 k^{2}-48 k-16344\right] \\
{[s, t, u, v]=[8 k-564,9 k-468,10 k-372,11 * k-276]} \\
{[w, x, y, z]=[-16 k-204,-15 k-108,-14 k-12,-13 k+84]} \\
\text { For } k=0 \text { we get }: \\
(a, b, c, d)=(7,-13,-93,227) \\
(s, t, u, v)=(47,39,31,23) \\
(w, x, y, z)=(17,9,1,-7) \\
7(47)^{3}+(-13)(39)^{3}+(-93)(31)^{3}+(227)(23)^{3}= \\
7(17)^{3}+(-13)(9)^{3}+(-93)(1)^{3}+(227)(-7)^{3}
\end{gathered}
$$




\section{Degree Four:}

$$
\begin{gathered}
a s^{4}+b t^{4}+c u^{4}+d v^{4}=a w^{4}+b x^{4}+c y^{4}+d z^{4} \\
\text { Let }(s, t, u, v)=(p m+1, q m+1, r m+1, m+1) \\
(w, x, y, z)=(p m-1, q m-1, r m-1, m-1)
\end{gathered}
$$

Substituting \& simplifying we get:

$$
\begin{gathered}
a\left(s^{4}-w^{4}\right)+b\left(t^{4}-x^{4}\right)+c\left(u^{4}-y^{4}\right)+d\left(v^{4}-z^{4}\right)=0 \\
\left(8 d+8 a p^{3}+8 b q^{3}+8 c r^{3}\right) m^{3}+(8 d+8 a p+8 b q+8 c r) m=0
\end{gathered}
$$

We get from above,

$$
\begin{gathered}
c=-\frac{a p^{3}+b q^{3}-a p-b q}{r\left(-1+r^{2}\right)} \\
d=\frac{-r^{2} a p+a p^{3}+b q^{3}-r^{2} b q}{\left(-1+r^{2}\right)}
\end{gathered}
$$

Next, find rational number $\{a, b, c, d, p, q, r\}$.

Let us use a known solution $[p, q, r]=[4,3,2]$,

Parametric solution:

$$
\begin{gathered}
(a, b, c, d)=\left(\begin{array}{c}
k^{2}-576 k+133120, k^{2}-576 k+133120 \\
k^{2}-576 k+133120,-27 k^{2}-576 k-1658880
\end{array}\right) \\
(s, t, u, v)=(2 k+1440, k+1280,1120, k-960) \\
(w, x, y, z)=(-6 k+160,-5 k+320,-4 k+480,-3 k+640)
\end{gathered}
$$

Numerical solution is:

$$
\begin{gathered}
(a, b, c, d)=(1,1,-14,21) \\
(s, t, u, v)=(9,7,5,3) \\
(w, x, y, z)=(7,5,3,1) \\
\text { Degree Five } \\
a s^{5}+b t^{5}+c u^{5}+d v^{5}=a w^{5}+b x^{5}+c y^{5}+d z^{5} \\
\text { Let }(s, t, u, v)=(p m+1, q m+1, r m+1, m+1) \\
(w, x, y, z)=(p m-1, q m-1, r m-1, m-1) \\
\text { Substituting \& simplifying we get: } \\
a\left(s^{5}-w^{5}\right)+b\left(t^{5}-x^{5}\right)+c\left(u^{5}-y^{5}\right)+d\left(v^{5}-z^{5}\right)=0 \\
\left(10 a p^{4}+10 b q^{4}+10 c r^{4}+10 d\right) m^{4} \\
+\left(20 a p^{2}+20 b q^{2}+20 c r^{2}+20 d\right) m^{2}+2 a+2 b+2 c+2 d=0 \\
\text { First, substitute d }=-a-b-c t o a b o v e \text { equation } . \\
\text { Next, find rational number }\{a, b, c, d, p, q, r, h\} . \\
\text { Let use a known solution }[p, q, r]=[4,3,2], \\
{[a, b, c, d]=[1,1,-9,7]} \\
h^{2}=-200\left(-1+p^{4}\right)\left(-1+p^{2}\right) a^{2}+ \\
{[a, b, c, d]=\left[k^{2}-800 k+1060000,\right.} \\
\left(\left(-200\left(-1+q^{4}\right)\left(-1+p^{2}\right)-200\left(-1+p^{4}\right)\left(-1+q^{2}\right)\right) b\right. \\
\left.-200\left(c r^{4}-c\right)\left(-1+p^{2}\right)-200\left(-1+p^{4}\right)\left(c r^{2}-c\right)\right) a \\
-200\left(-1+q^{4}\right)\left(-1+q^{2}\right) b^{2}+\left(-200\left(c r^{4}-c\right)\left(-1+q^{2}\right)\right. \\
\left.-200\left(-1+q^{4}\right)\left(c r^{2}-c\right)\right) b-200\left(c r^{4}-c\right)\left(c r^{2}-c\right) \\
V=400
\end{gathered}
$$




$$
\begin{gathered}
k^{2}-800 k+1060000,-9 k^{2}-800 k-17140000 \\
\left.7 k^{2}+2400 k+15020000\right] \\
{[s, t, u, v]=[k-13900,2 k-11300,3 k-8700,4 k-6100]} \\
{[w, x, y, z]=[-9 k-6900,-8 k-4300,-7 k-1700,-6 k+900]} \\
\text { Degree six: } \\
a s^{6}+b t^{6}+c u^{6}+d v^{6}=a w^{6}+b x^{6}+c y^{6}+d z^{6} \\
\text { Let }(s, t, u, v)=(p m+1, q m+1, r m+1, m+1) \\
(w, x, y, z)=(p m-1, q m-1, r m-1, m-1) \\
\text { Substituting \& simplifying we get: } \\
a\left(s^{6}-w^{6}\right)+b\left(t^{6}-x^{6}\right)+c\left(u^{6}-y^{6}\right)+d\left(v^{6}-z^{6}\right)=0 \\
\left(12 a p^{5}+12 d+12 c r^{5}+12 b q^{5}\right) m^{5} \\
+\left(40 b q^{3}+40 a p^{3}+40 d+40 c r^{3}\right) m^{3}+(12 c r+12 b q+12 a p+12 d) m=0
\end{gathered}
$$

First, substitute $d=-c r-b q-$ ap to above equation.

Next, find rational number $\{a, b, c, d, p, q, r\}$

Let use a known solution $[p, q, r]=[4,3,2]$,

$$
[a, b, c, d]=[1,1,-18,29]
$$

\section{Parametric Solution:}

$$
(a, b, c, d)=\left(k^{2}-5760 k+39484800,\right.
$$

$k^{2}-5760 k+39484800,-18 k^{2}-5760 k-1019347200$,

$$
\left.29 k^{2}+51840 k+1762300800\right)
$$

$(s, t, u, v)=([k+58680,47880, k-37080,2 k-26280])$

$(w, x, y, z)=([7 k+27720,6 k+16920,5 k+6120,4 k-4680])$

Degree Seven:

$$
\begin{gathered}
a s^{7}+b t^{7}+c u^{7}+d v^{7}=a w^{7}+b x^{7}+c y^{7}+d z^{7} \\
\text { Let }(s, t, u, v)=(p m+1, q m+1, r m+1, m+1) \\
(w, x, y, z)=(p m-1, q m-1, r m-1, m-1)
\end{gathered}
$$

Substituting \& simplifying we get:

$$
\begin{gathered}
a\left(s^{7}-w^{7}\right)+b\left(t^{7}-x^{7}\right)+c\left(u^{7}-y^{7}\right)+d\left(v^{7}-z^{7}\right)=0 \\
\left(14 a p^{6}+14 d+14 c r^{6}+14 b q^{6}\right) m^{6} \\
+\left(70 b q^{4}+70 a p^{4}+70 d+70 c r^{4}\right) m^{4} \\
+\left(42 c r^{2}+42 b q^{2}+42 a p^{2}+42 d\right) m^{2} \\
+2(a+b+c+d)=0
\end{gathered}
$$

We take $(c, d)$ as shown below, we also have $d=-(a+b+c)$

$$
\begin{gathered}
c=-\frac{b q^{2}+a p^{2}-a-b}{r^{2}-1} \\
d=\frac{-r^{2} a-r^{2} b+b q^{2}+a p^{2}}{r^{2}-1}
\end{gathered}
$$

Hence we have to find rational number $\{a, b, c, d, p, q, r\}$.

Example: $[p, q, r]=[5,4,2],[a, b, c, d]$

$$
=[11,-35,87,-63]
$$




$$
\begin{gathered}
h^{2}=-980\left(r^{2}-r^{2} p^{2}+p^{4}-p^{2}\right)\left(r^{4}-r^{4} p^{2}+r^{2}-r^{2} p^{2}+p^{6}-p^{2}\right) a^{2} \\
+\left(-980\left(r^{2}-r^{2} q^{2}+q^{4}-q^{2}\right)\left(r^{4}-r^{4} p^{2}+r^{2}-r^{2} p^{2}+p^{6}-p^{2}\right)\right. \\
\left.-980\left(r^{2}-r^{2} p^{2}+p^{4}-p^{2}\right)\left(r^{4}-r^{4} q^{2}+r^{2}-r^{2} q^{2}+q^{6}-q^{2}\right)\right) b a \\
-980\left(r^{2}-r^{2} q^{2}+q^{4}-q^{2}\right)\left(r^{4}-r^{4} q^{2}+r^{2}-r^{2} q^{2}+q^{6}-q^{2}\right) b^{2}
\end{gathered}
$$

Thus it is sufficient to solve the quadratic

equation for $\{a, b, h\}$.

We get a parametric solution

$$
a=11 k^{2}-317520 k+130557873600
$$$$
b=-35 k^{2}-317520 k-452218334400
$$$$
c=87 k^{2}+4127760 k+1216628683200
$$$$
d=-63 k^{2}-3492720 k-894968222400
$$

$$
\begin{gathered}
{[s, t, u, v]=[-2 k-982800,-k-839160, k-551880,2 k-408240]} \\
{[w, x, y, z]=[-8 k-453600,-7 k-309960,} \\
-5 k-22680,-4 k+120960] \\
k \text { is arbitrary. }
\end{gathered}
$$

\section{Degree Eight:}

$$
\begin{gathered}
a s^{8}+b t^{8}+c u^{8}+d v^{8}=a w^{8}+b x^{8}+c y^{8}+d z^{8} \\
\text { Let }(s, t, u, v)=(p m+1, q m+1, r m+1, m+1) \\
(w, x, y, z)=(p m-1, q m-1, r m-1, m-1)
\end{gathered}
$$

Substituting \& simplifying we get:

$$
a\left(s^{8}-w^{8}\right)+b\left(t^{8}-x^{8}\right)+c\left(u^{8}-y^{8}\right)+d\left(v^{8}-z^{8}\right)=0
$$

In a similar way:

we have to find rational number $\{a, b, c, d, p, q, r\}$.

We have known solution: $[p, q, r]=[4,3,2]$,

$$
[a, b, c, d]=[1,-7,18,-19]
$$

Parametric solution is:

$$
\begin{gathered}
a=k^{2}-53760 k+23843635200 \\
b=-7 k^{2}-53760 k-178465996800 \\
c=18 k^{2}+752640 k+475427635200 \\
d=-19 k^{2}-1128960 k-510831820800
\end{gathered}
$$

$[s, t, u, v]=[-2 k-1021440,-k-833280,-645120, k-456960]$

$[w, x, y, z]=[-6 n-483840,-5 n-295680,-4 n-107520,-3 n+80640]$

\section{REFERENCES}

[1] Ajai Choudhry, Diophantine equation, for fifth degree, Rocky mountain journal of mathematics, vol.29,no.2, (1999)

[2] Oliver Couto, Published Math paper, Taxicab Equations for power (2, 3, 4 \& 5), Journal, International Math Forum, Hikari ltd., Vol.9, Jan 2014.n0.12, pages 561-577.

[3] Oliver Couto \& Seiji Tomita, Solution of Polynomial Equation of any Degree ' $n$ ' with Special Emphasis for $n=(2,3,4,5 \& 6)$, Journal "SOP transactions on applied mathematics" ISSN(Print): 2373-8472 ISSN: 2373-8480, July 2015

[4] Seiji Tomita, Computation number theory web pages: http://www.maroon.dti.ne.jp/fermat 
[5] Oliver Couto, Web page on Mathematics, http://www.celebrating-mathematics.com

[6] Seiji Tomita, Fourth power polynomial equation, Computation number theory-webpage, http://www.maroon.dti.ne.jp/fermat/diop122e.html

[7] Seiji Tomita, sixth power polynomial equation, Computation number theory- Web-page, http://www.maroon.dti.ne.jp/fermat/diop160e.html

[8] Ramanujan lost notebook, Narosa publishing house

[9] Euler Leonhard, Opera Omnia, 1984

[10] Tito Piezas-Online collection of algebraic identities http://sites.google.com/site/tpiezas

[11] Ajai Choudhry, Symmetrical Diophantine equations, Journal of mathematics, Rocky mountain journal vol. 34,no.4,winter(2004), pg. 1261-1298.

[12] Jaroslaw Wroblewski, Tables of Numerical solutions for degree three, four, six seven \& nine, website, www.math.uni.wroc.pl/ jwr/eslp

[13] L. E. Dickson, history of the theory of numbers, Vol.II, (Diophantine analysis), AMS Chelsea publication, reprinted year 2000) 